\title{
Development of photonic crystal structures for on-board optical communication
}

\author{
Muhammad Umar Khan*a, John Justice ${ }^{\mathrm{a}}$, Arjen Boersma ${ }^{\mathrm{b}}$, Maurice Mourad ${ }^{\mathrm{b}}$, Renz van Ee ${ }^{\mathrm{b}}$, Alfons \\ van Blaaderen ${ }^{\mathrm{c}}$, Judith Wijnhoven ${ }^{\mathrm{c}}$, Brian Corbett ${ }^{\mathrm{a}}$ \\ ${ }^{\mathrm{a}}$ Tyndall National Institute, Lee Maltings, Prospect Row, Cork, Ireland; ${ }^{\mathrm{b}} \mathrm{TNO}$, De Rondom 1 \\ 5612AP, Eindhoven, The Netherlands; ${ }^{\mathrm{c}}$ Soft Condensed Matter, Ornstein Laboratory, Utrecht, The \\ Netherlands.
}

\begin{abstract}
We present designs for sharp bends in polymer waveguides using colloidal photonic crystal $(\mathrm{PhC})$ structures. Both silica $\left(\mathrm{SiO}_{2}\right)$ sphere based colloidal $\mathrm{PhC}$ and core-shell colloidal $\mathrm{PhC}$ structures having a titania $\left(\mathrm{TiO}_{2}\right)$ core inside silica $\left(\mathrm{SiO}_{2}\right)$ shells are simulated. The simulation results show that core-shell Face Centered Cubic (FCC) colloidal crystals have a sufficient refractive index contrast to open up a bandgap in the desired direction when integrated into polymer waveguides and can achieve reflection $>70 \%$ for the appropriate plane. Different crystal planes of the FCC structure are investigated for their reflection and compared with the calculated bandstructure. Different techniques for fabrication of $\mathrm{PhC}$ on rectangular seed layers namely slow sedimentation; spin coating and modified doctor blading are discussed and investigated. FCC and Random FCC silica structures are characterized optically to show realisation of (001) FCC.
\end{abstract}

Keywords: Photonic Crystal, Silica, Face Centre Cubic, Core-shell, Bandstructure, Doctor blading, Sedimentation, Spin coating.

\section{INTRODUCTION}

Single mode polymer waveguides can be used to guide information-carrying light from one component to another on a backplane. The low cost and ease of fabrication associated with polymer waveguides makes them attractive but the low index contrast inherent in polymeric materials requires large bending radii to reduce radiation loss making it difficult to achieve a high component density. Thus, a means to have more compact bends in polymer optical waveguides is required. Photonic crystals are artificial periodic structures which have been used to manipulate the properties of light. Some applications for photonic crystal are to confine/trap light at resonance ${ }^{1}$, compress a light pulse by slowing it down ${ }^{2}$, split light into different polarizations ${ }^{3}$, optical trapping ${ }^{4}$ and to enhance nonlinear effects ${ }^{5}$. Periodicity and refractive index contrast are the main parameters that determine the functionality of these structures. In this paper, we present the design and fabrication of photonic crystal structures that can act as an in-plane reflector for polymer waveguides at wavelengths in the $1550 \mathrm{~nm}$ range as used in telecommunications.

We have designed the different photonic crystal structures to be integrated with $5 \mu \mathrm{m}$ wide polymer waveguides. The photonic crystal structures need to be of at least the same size as that of the waveguide for efficient bending of the light. Thus, the mature two dimensional photonic crystal structures cannot be used. Rather, three dimensional structures need to be implemented. The optical response of the different structures are simulated and compared where we use the commercially available Lumerical Solutions software based on the Finite Difference Time Domain (FDTD) method.

Natural sedimentation of mono-dispersed particles on a flat surface is the easiest way to realise three dimensional photonic crystal structures. The multi-layer structures fabricated in this manner can be expected to have horizontal layers of hexagonally packed spheres stacked above each other. The Face Centre Cubic (FCC) structure is formed only if the exact positioning of each individual horizontal layer of spheres repeats itself at every third layer in vertical direction. Three different positioning, repeating every third layer, can be designated as A, B and C arrangements and the stacking of repeated ABCABC... is the FCC stacking as shown in Fig. 1. The FCC lattice is formed only if everything goes right in the natural sedimentation process with the spheres positioning themselves at the right locations

Photonic Crystal Materials and Devices XI, edited by Sergei G. Romanov, Gabriel Lozano,

Dario Gerace, Christelle Monat, Hernán Ruy Míguez, Proc. of SPIE Vol. 9127, 912705

(C) 2014 SPIE · CCC code: 0277-786X/14/\$18 - doi: 10.1117/12.2052214

Proc. of SPIE Vol. 9127 912705-1 
without any defects. Defects in the sedimentation process can change the repetition from say the third to every second layer resulting in $\mathrm{AB}, \mathrm{BC}$ or $\mathrm{AC}$ stacking. Structures having alternating layers of spheres like $\mathrm{ABAB}$...are called Hexagonal Closed Pack (HCP) structures. So, any deviation from ideal sedimentation will result in changing of FCC into HCP and a mix of FCC and HCP structures results. This mix of FCC and HCP structures can be named as a Random Face Centered Cubic (RFCC) structure. In this paper, we propose to realise FCC structures using a starting surface with a rectangular seed layer which may be less prone to defect generation. FCC structures fabricated using both natural sedimentation and the new proposed technique are simulated to show that pure FCC structures having sufficient index contrast can be used to achieve in-plane bending of light at sharp angles.

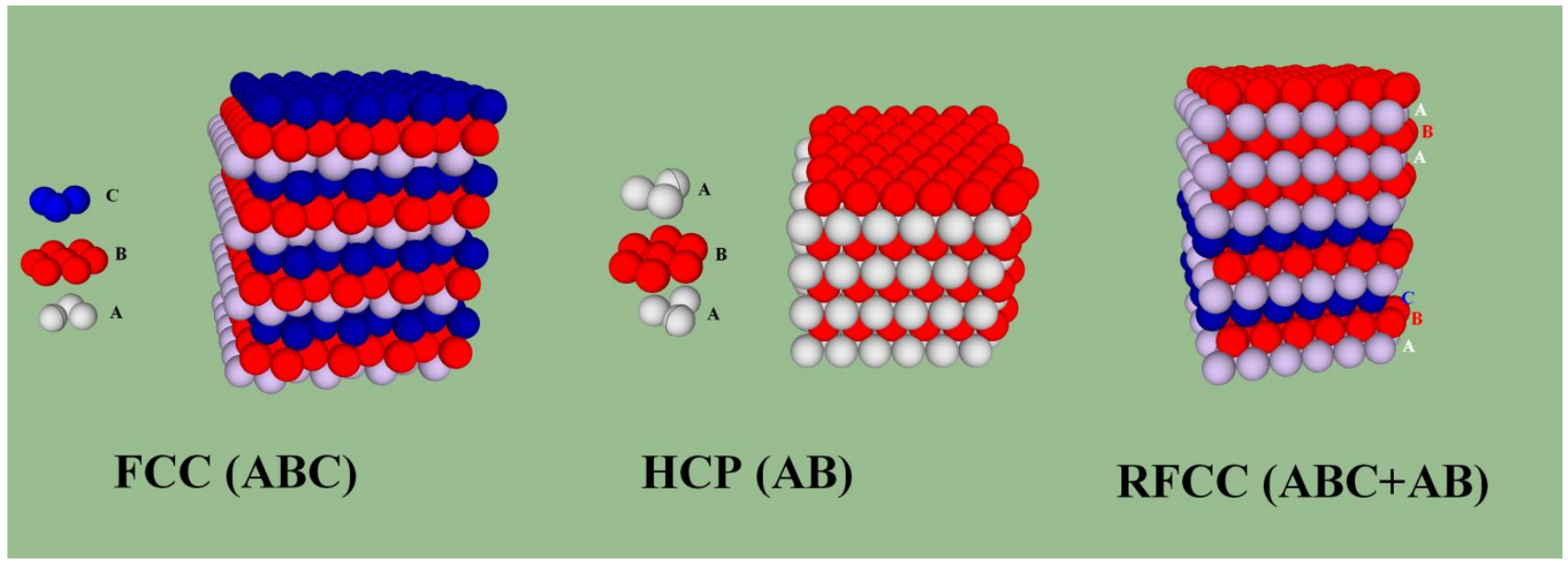

Fig. 1: Three different relative arrangements of spheres: $\mathrm{A}, \mathrm{B}$ and $\mathrm{C}$ are represented in White, Red and Blue colors respectively to show the stacking of Face Centered Cubic (FCC), Hexagonal Closed Packed (HCP) and Random Face Centered Cubic (RFCC) structures.

\section{FCC STRUCTURES FOR IN-PLANE BENDING}

The optical properties of a FCC structure with $1000 \mathrm{~nm}$ diameter silica spheres is modelled and simulated. The photonic crystal structure is 'integrated' at the end of a $5 \mu \mathrm{m}$ high polymer waveguide with the intention to bend the light travelling inside the waveguide. The FCC structure of silica spheres will act as a reflector if the index contrast $(\Delta \mathrm{n})$ between the silica spheres $\left(\mathrm{n}_{\text {silica }}=1.45\right)$ and the background air $\left(\mathrm{n}_{\text {air }}=1.0\right)$ is sufficient to open up a band-gap at the incident direction. In our example, the photonic crystal is tilted at $45^{\circ}$ to the incident light in order to bend the light at $90^{\circ}$. The reflected light will be guided by a polymer waveguide placed at a right angle to the incident waveguide. In the simulations, air acts as the cladding of the waveguides and background for the stacked silica spheres. The modelled photonic crystal structure with the waveguide arrangement and the calculated $90^{\circ}$ and back reflected optical powers are shown in Fig. 2. 

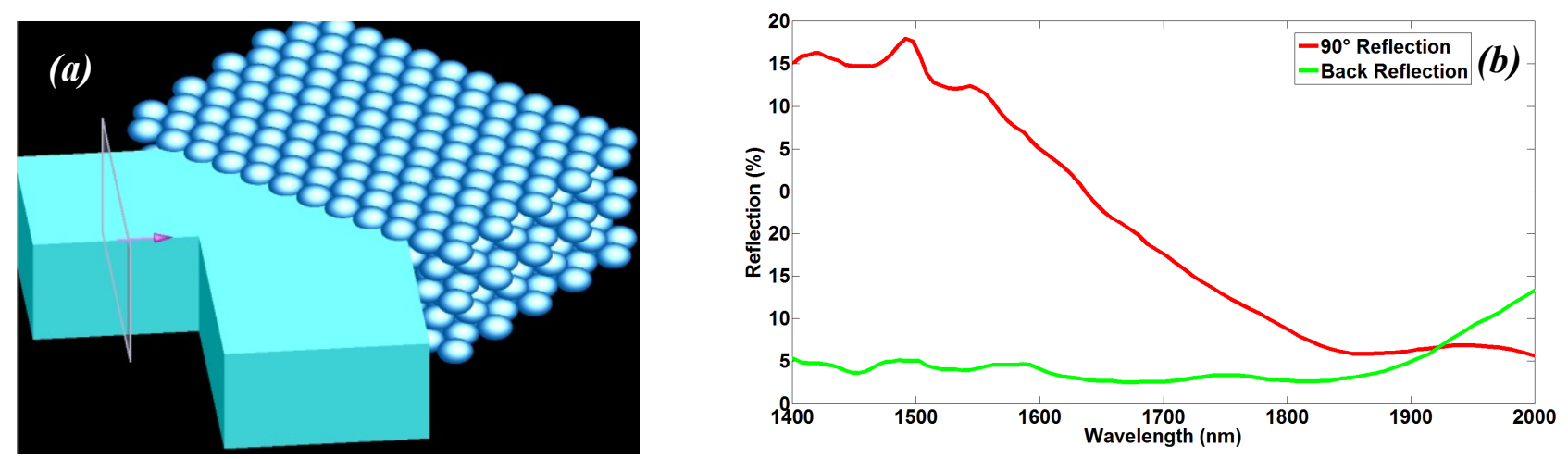

Fig. 2: (a) Modelled natural sedimentation FCC photonic crystal structure embedded in polymer waveguides for $90^{\circ}$ bending of light. (b) $90^{\circ}$ and back reflected optical powers are plotted in red and green colors respectively.

Reflections from the structure can be expected at a wavelength of $1500 \mathrm{~nm}$ using Bragg's Law for an effective refractive index of $n_{e f f}=1.33$. The effective refractive index of the FCC structure is calculated from $n_{\text {eff }}=n_{\text {silica }} *$ $0.74+n_{\text {air }} * 0.26$ where 0.74 is the packing factor of the silica spheres in air. The red plot in Fig. 2(b) confirms $90^{\circ}$ reflections around $1500 \mathrm{~nm}$. The small reflection intensity shows that the index contrast between silica and air is not sufficient to open up a wide bandgap which results in most of the light transmitting through the structure. The green plot in Fig. 2(b) shows negligible reflections in the backward direction.

A larger refractive index contrast is required for higher reflectivity and can this be achieved by increasing refractive index of the spheres. Core-shell spheres having core of higher refractive index material $\mathrm{TiO}_{2}\left(\mathrm{n}_{\text {titania }}=2.4\right)$ inside a silica $\left(\mathrm{n}_{\text {silica }}=1.45\right)$ shell can be considered. Such core-shell particles embedded into polymer waveguides are modelled and simulated to see whether the index contrast is sufficient for such structures to be efficient reflectors at telecom wavelengths. The effective refractive index of the core-shell particles having $n_{\text {core }}=2.4$ and $n_{\text {shell }}=1.45$ is calculated to be $n_{\text {sphere }}=1.67$ using $n_{\text {sphere }}=\left[n_{\text {core }} * \frac{4}{3} \pi r_{\text {core }}^{3}\right]+\left[n_{\text {shell }} * \frac{4}{3} \pi\left(r_{\text {shell }}^{3}-r_{\text {core }}^{3}\right)\right]$. The effective refractive index of core-shell FCC structure in air background is calculated to be 1.5 using $n_{\text {eff }}=0.74 * n_{\text {sphere }}+0.26 *$ $n_{\text {background }}=1.5$ where 0.74 is the sphere packing factor in FCC. The larger refractive index contrast $\Delta n=0.67$ between the core-shell particles and the background air is very helpful for the photonic crystal to act as a reflector. The calculated reflection intensities from core-shell particles crystal are shown in Fig. 3. The red plot shows that more than $50 \%$ reflection at $90^{\circ}$ can be obtained around telecom wavelengths. These reflections from photonic crystal reflector also follow Bragg's law and move to smaller wavelengths with an increase in angle of incidence.

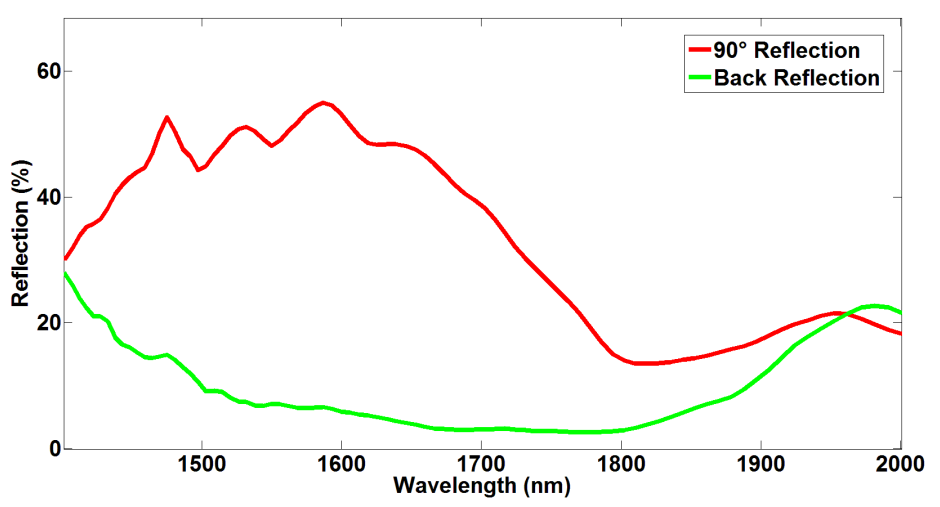

Fig. 3: Red plot shows calculated optical power in $90^{\circ}$ waveguide. Green plot shows calculated optical power reflected backwards in the incident waveguide from $\mathrm{TiO}_{2}-\mathrm{SiO}_{2}$ core-shell photonic crystal structure.

The possibility of forming RFCC structures using the natural sedimentation process will make it difficult to obtain a pure FCC structure. This lack of control in stacking might be overcome if the spheres are forced to position 
themselves at some particular points. Any pits or pillars on an underlying surface can force the spheres to sit on the desired locations which could ultimately result in better stacking of the spheres. We propose using a rectangular seed layer, in which spheres are stacked over a rectangular grid of small holes as shown in Fig. 8(B). The FCC (001) plane is shown in Fig. 8(B) with the base of the FCC unit cell identified. This seed layer is intended to force the spheres to arrange themselves in a rectangular arrangement in contrast to a hexagonal arrangement without the seed layer. The subsequent layer of spheres will fit themselves in the grooves formed from first layer of spheres and thus should help to make a well stacked rectangular seed layer structure.

A three dimensional sphere structure based on a rectangular seed layer is depicted in Fig. 4. The base of the FCC unit cell and the selected FCC reflecting plane are marked in black and red colors respectively. The structure in Fig. 4(a) shows that FCC (001) reflecting plane is at $45^{\circ}$ to the incident light which can only be exposed directly to the incoming light if the redundant spheres indicated by the yellow triangle are removed. These spheres in front of the selected reflecting plane decrease the reflection efficiency due to them scattering the light in different directions. Thus we truncate the photonic crystal structure at $45^{\circ}$ to access the desired FCC plane as is shown in Fig. 4(b). Core-shell particle structures fabricated on rectangular seed layer are simulated to show these structures can be used for in-plane bending of light. Truncated core-shell structures are simulated to calculate amount of optical power reflecting at $90^{\circ}$ to the incident light. Simulations show that more than $70 \%$ of incident light can be reflected from truncated structure as shown by the plot in Fig. 5(a). Truncating the structure makes FCC (001) plane at $45^{\circ}$ to the incident accessible which results in better efficiency.

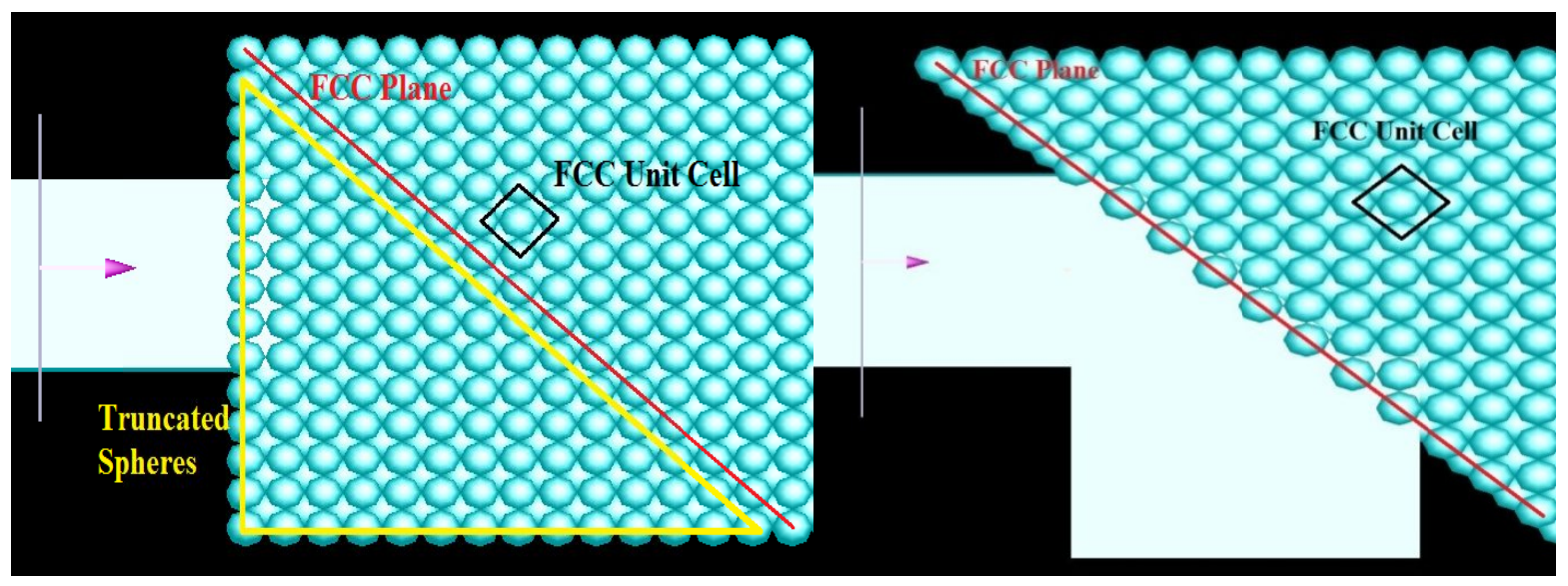

(a)

(b)

Fig. 4: (a) Rectangular seed layer structure showing FCC unit cell, FCC reflecting plane and the excess spheres to be truncated in black, red and yellow colors respectively. (b) Structure is truncated at $45^{\circ}$ to expose appropriate FCC plane to the incident light.

The calculated bandstructure of the FCC crystal is shown in Fig. 5(b). The bandstructure shows openings of band-gaps in different orientations of the crystal lattice in reciprocal space. The calculated reflection from the (111) plane is shown in Fig. 6(a). The reflection spectrum is plotted on top of the bandstructure in Fig. 6(b) to show that (111) plane of FCC is represented by $\Gamma$-L direction in reciprocal space. The reflection peak from the (111) plane falls exactly on top of the band-gap with Full Width Half Maximum (FWHM) equal to the opening of the band-gap. The bandstructure dispersion in the $\Gamma$ - $\mathrm{L}$ direction is linear and behaves like a simple Bragg reflector. This band-gap in $\Gamma$ - $\mathrm{L}$ direction moves to higher frequencies as we move from $\mathrm{L}$ to $\mathrm{W}$ directions in the reciprocal space. Movement from $\mathrm{L}$ to $\mathrm{W}$ points in the reciprocal space is equivalent to changing the angle of incidence of light on (111) plane. This movement of band-gap to higher frequencies is in agreement with the Bragg's law of dispersion with the resonance frequency moving to larger values with an increase in the angle of incidence. 


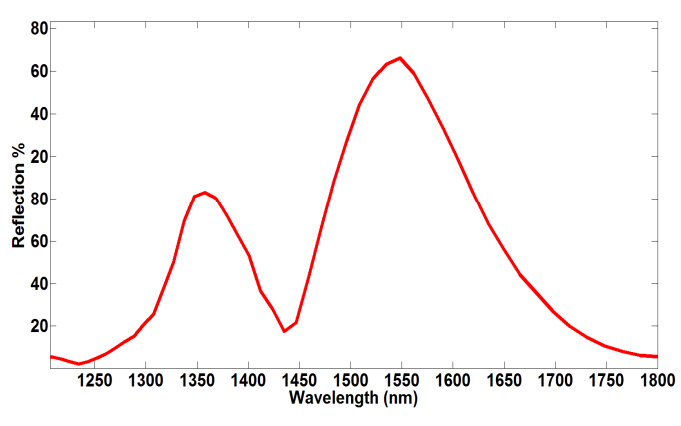

(a)

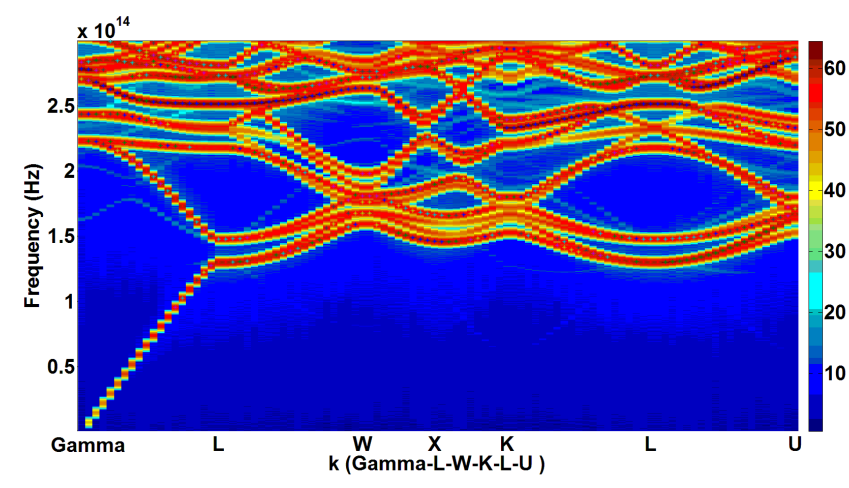

(b)

Fig. 5: (a) $90^{\circ}$ reflection response from truncated rectangular seed layer structure. (b) Bandstructure of core-shell FCC crystal.

Fig. 5(b) shows that band-gap in L-U and L-K directions follow the same trend so it can be concluded that L-U and L-K directions in the reciprocal space are identical. Reflections from (001) plane of FCC unit cell is mapped to the middle point of $\mathrm{W}-\mathrm{K}$ directions which is named as $\mathrm{X}$. The calculated reflection response for normal incidence of light on (001) plane of core-shell FCC photonic crystal shows a reflection peak around 1.6 THz. The reflection spectrum from the (001) plane of core-shell FCC is plotted on top of the calculated bandstructure in Fig. 7(a) to show that reflection peak falls in the band-gap frequencies at $\mathrm{X}$ point which is right in the middle of $\mathrm{W}-\mathrm{K}$ direction. It can be observed in the bandstructure that band-gap at X moves to larger frequency values on moving towards $\mathrm{W}$ or $\mathrm{K}$ point from $\mathrm{X}$.

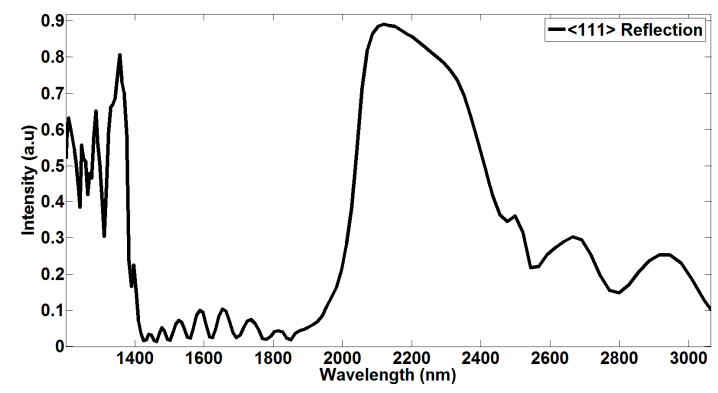

(a)

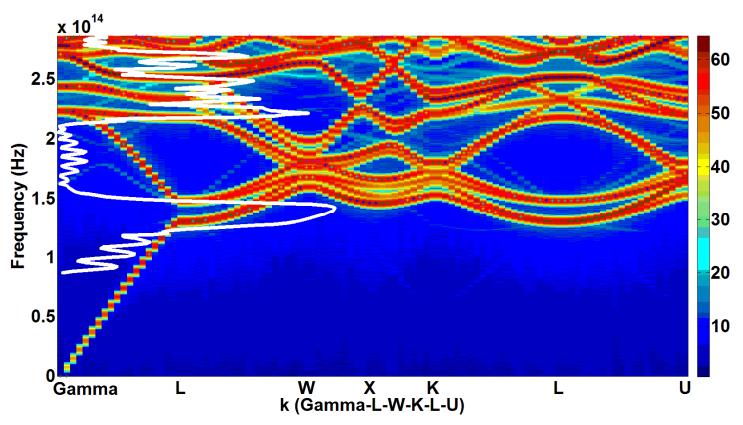

(b)

Fig. 6: (a) Calculated reflection spectrum at normal incidence to (111) plane of the FCC crystal. (b) Reflection spectrum from (111) FCC plane plotted against bandstructure showing band-gap in the $\Gamma$-L direction.

Fig. 5(b) shows inter-crossings of optical bands near K/U and $\mathrm{W}$ points in the bandstructure. The crossings of the bands result in transfer of energy between the optical bands present for those frequencies. A small reflection peak in the reflection response appears just next to the strong reflection peak at the start of the intercrossing. This new peak gets energy from the stronger peak in the neighborhood in result of the energy transfer. As a result, the initial peak before the start of the crossing transfers its complete energy to the new born peak and disappears. This generation of two peaks in the reflection response because of the intercrossing can be observed on changing the angle of incidence on (001) plane of FCC. The relatively small peak next to the larger reflection peak in Fig. 5(a) for $45^{\circ}$ incidence of light on truncated coreshell FCC is because of the intercrossing of bands. Reflection response for $45^{\circ}$ incidence on (001) plane of FCC is plotted on the bandstructure in Fig. 7(b) to show the agreement between the bandstructure and calculated reflection spectrum. It can be observed in Fig. 7(b) that the reflection peak for $45^{\circ}$ incidence is situated at larger values of frequencies which is as per predictions from the bandstructure. Two peaks at $1.6 \mathrm{THz}$ and $1.85 \mathrm{THz}$ are observed 
because of the inter-crossings. It is observed that the peak at $1.85 \mathrm{THz}$ just next to the initial peak at $1.6 \mathrm{THz}$ gets stronger with increasing angle of incidence and depletes the $1.6 \mathrm{THz}$ peak.

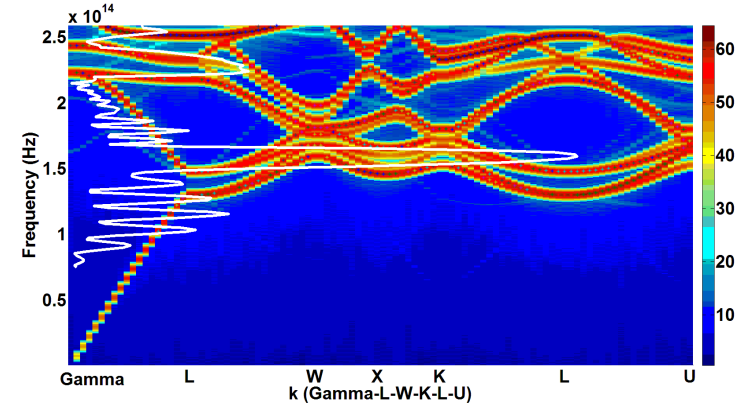

(a)

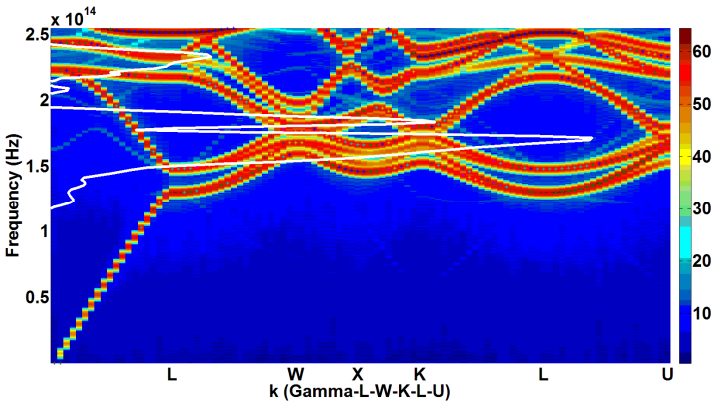

(b)

Fig. 7: (a) Reflection spectrum from (001) FCC plane is in agreement with band-gap at X point (b) Reflection response from (001) FCC plane at $45^{\circ}$ incidence shows two peaks because bands are inter-crossing at that point.

\section{MANUFACTURING AND CHARACTERIZATION OF COLLOIDAL CRYSTALS}

The crystal structure and orientation of the particles in the colloidal crystals determine the reflection and transmission of the light and thus the properties of the photonic crystal. The preferred crystal form of mono-dispersed particles is the FCC stacking. Fabrication of FCC structures without any external field or force will result in a hexagonal ground plane as shown in Fig. 8(A). The presented hexagonal plane is the (111) plane of the FCC structure. It has been shown by A. Mihi et $\mathrm{al}^{6}, \mathrm{H}$. Yang et $\mathrm{al}^{7}$ and Y. L. $\mathrm{Wu}^{8}$ that application of external forces during spincoating or doctor blading can result in the formation of an FCC with different orientations than (111) FCC. Here, we have selected a rectangular ground plane i.e. (001) plane of FCC instead of (111) plane as shown in Fig. 8(B).

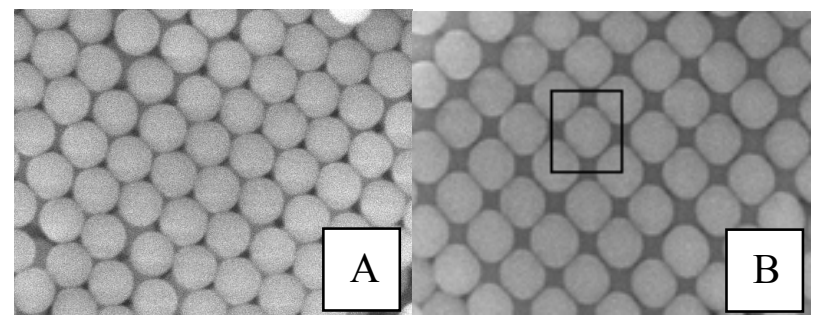

Fig. 8: (A) (111) FCC plane. (B) (001) FCC plane with marked base of FCC cell.

The rectangular ground plane stacking has two major advantages over the hexagonal ground layer for the formation of photonic crystals. Starting from a square ground plane, only one type of crystal should be formed in the subsequent layers i.e. FCC, and all these layers form reflection planes perpendicular to the substrate. Unfortunately, the manufacturing of (001) FCC using only external forces is very difficult to control. Crystal regions are formed in small domains throughout a large sample, and the positioning of such crystals cannot be done accurately with respect to any optical features, such as waveguides. In order to overcome this issue, we used a template having a square array of holes to force the colloid to crystallize in the (001) FCC layer ${ }^{9}$, which should induce self-assembly of the subsequent layer into the (001) FCC crystals. The template is manufactured by nano-imprint lithography. The nano-imprint stamp has an array of rods which is marked on to a polymer substrate. During imprinting, the polymer is solidified around the rods using UV light. In our experiments, this results in the formation of an area of $1 \mathrm{~mm}^{2}$ having holes at a distance of 900 to 1100 $\mathrm{nm}$. This variation was used in order to assess the influence of the pitch on the crystal formation. Different methods of growth can be used to fabricate square lattice structures over nano-imprinted seed layer region. We have used spin coating, slow sedimentation and modified doctor blading which are shown in Fig. 9 A, B and C respectively. 
Fig. 9(A) shows the process of spin coating over a rectangular seed layer region. A diluted solution of monodisperse spheres is spun on the seed layer region at a desired rotation speed. The rotation speed of the spinner defines the thickness of the fabricated sample so a sample with larger number of layers can be fabricated using a lower number of rotations per minute. Fig. 9(B) shows the slow sedimentation process in which a diluted solution of the spheres is placed on top of seed layer region for substantial amount of time to let the spheres sediment on the seed layer. The thickness of the sample depends on the concentration of the sample and the amount of time the spheres are allowed to sediment. Fig. $9(\mathrm{C})$ shows the modified doctor blading process in which spheres are spread over the seed layer region using doctor blade machine.

A

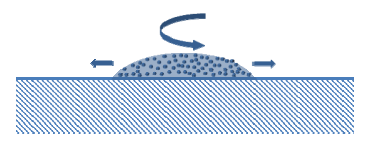

$\mathrm{B}$

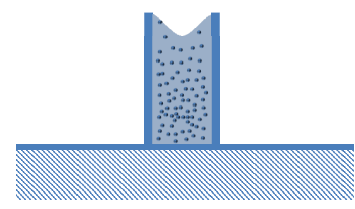

$\mathrm{C}$

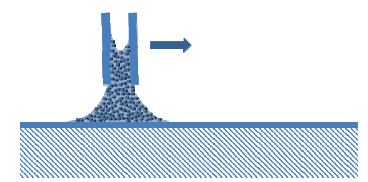

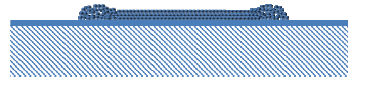
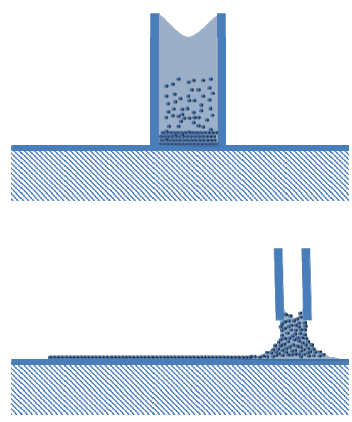

Fig. 9: Manufacturing process of colloidal crystal on ground plane having a square array of holes.

It is found that size of the particles should match the pitch size of the seed layer for a good stacking of the particles. Different pitch sizes compared with the size of the particles results in random stacking without any orientation. This can be seen in Fig. 10(a) and Fig. 10(b) where sphere sizes are smaller and bigger than the pitch sizes respectively.

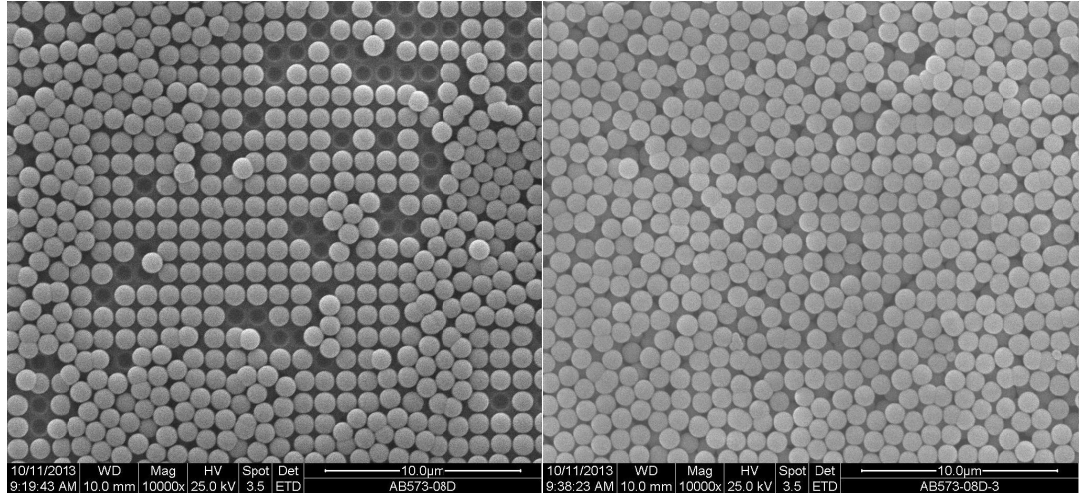

(a) (b)

Fig. 10: One and two layers of $1000 \mathrm{~nm}$ silica particles on a square imprinted seed layer made by the doctor blading approach. (a) pitch is $1100 \mathrm{~nm}$. (b) pitch is $980 \mathrm{~nm}$.

The first multilayer (001) FCC stacking was performed using the slow sedimentation process of $1000 \mathrm{~nm}$ silica spheres from a diluted $(0.1 \mathrm{wt} \%)$ dispersion of particles in a mixture of water/ethanol (1:1). After 20 hours a stack of approximate 7 to 8 layers was formed on the imprinted seed layers. A combination of both (001) and (111) planes on the top layer was observed. (001) planes were observed only in the seed layer regions as opposed to (111) planes on top of regions without seed layer. It is observed that some regions having seed layer underneath also have a mix of (111) and (001) lattice. Fig. 11 shows the top layer of a sedimentation sample on the seed layer, which is a combination of (001) and (111) FCC crystals. 


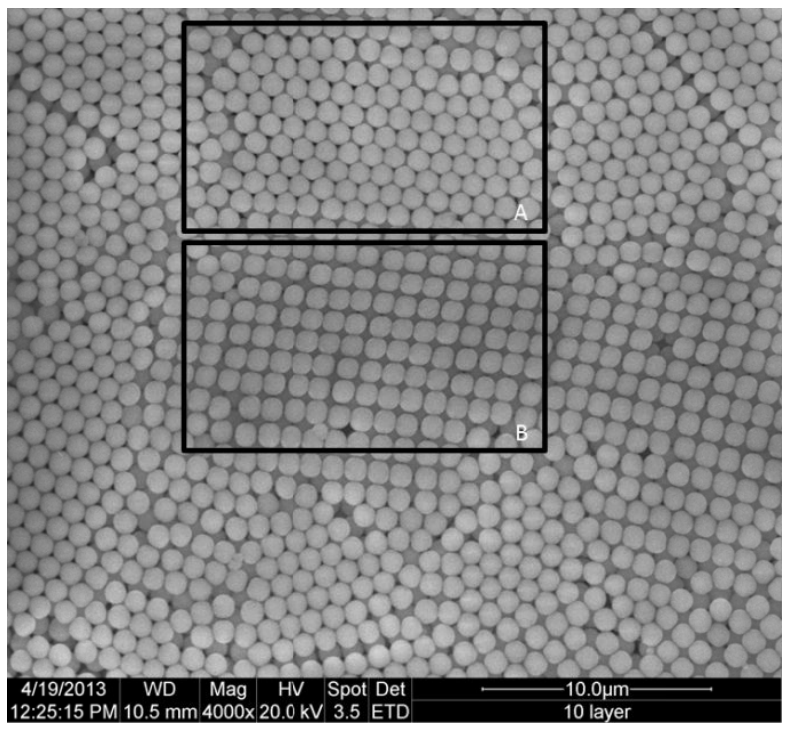

Fig. 11: Sedimentation of $1000 \mathrm{~nm}$ silica spheres on square seed layer in A - (111) FCC, and B - (001) FCC stacking.

In order to assess the number of layers, confocal laser scanning fluorescent microscopy was performed. The $1^{\text {st }}$ and $8^{\text {th }}$ layer is shown in Fig. 12. The first layer is almost perfectly square, the eighth layer shows several cracks, due to drying of the sample. Furthermore, a lot of the square stacking is lost, but some areas still show the (001) FCC stacking which show that stacking can be improved with some improvements in the fabrication process.
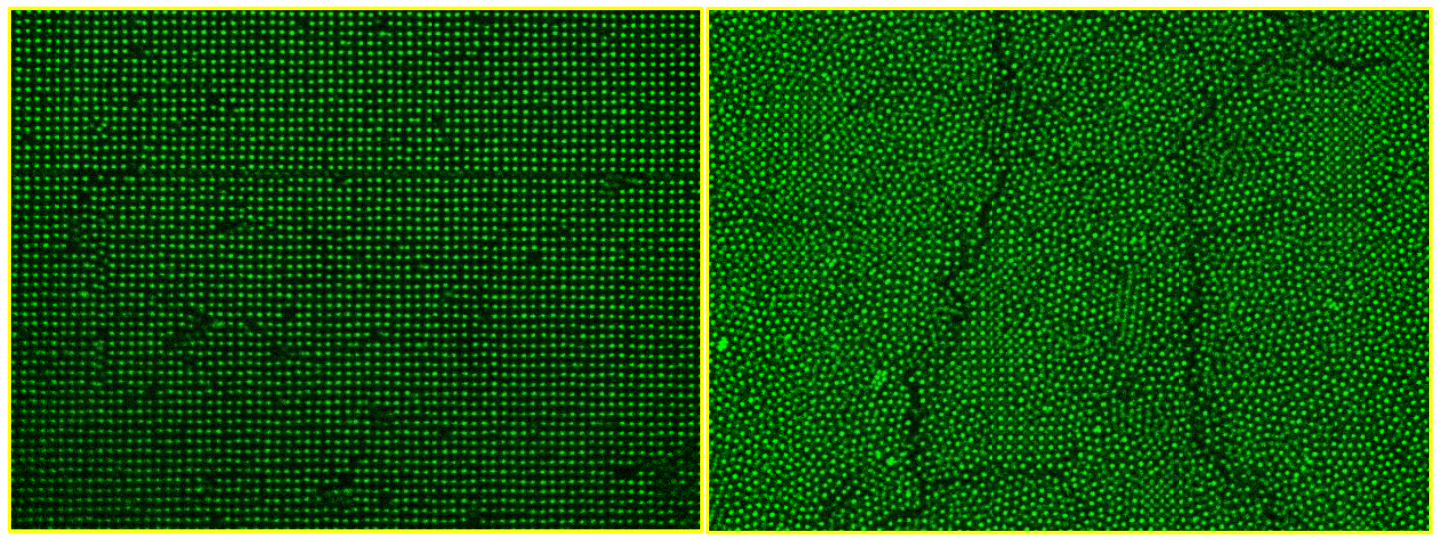

Fig. 12: First and eighth layer of a sedimentation sample of $900 \mathrm{~nm}$ silica spheres on a $940 \mathrm{~nm}$ pitch seed layer.

These fabricated silica sphere crystals are characterized optically to observe the reflections. Broadband white light from a white light source is vertically incident on the sample to measure the back reflection from the sample. Light is incident from an optical circulator which couples the back reflections from the sample to fiber port connected to an optical spectrometer. It is observed that reflection from the sample is position dependent. Different reflection spectra are observed from different regions of the sample. Careful investigation has revealed that two different responses are being observed from the regions having (001) and (111) regions as shown in figure below.At one position, the RFCC reflection can be seen, but at other positions the (001) FCC reflection is measured. This indicates that the seed layer indeed induces the formation of the FCC crystal in the correct orientation. Further, optimization will be required to control the sizes of these crystal domains. 


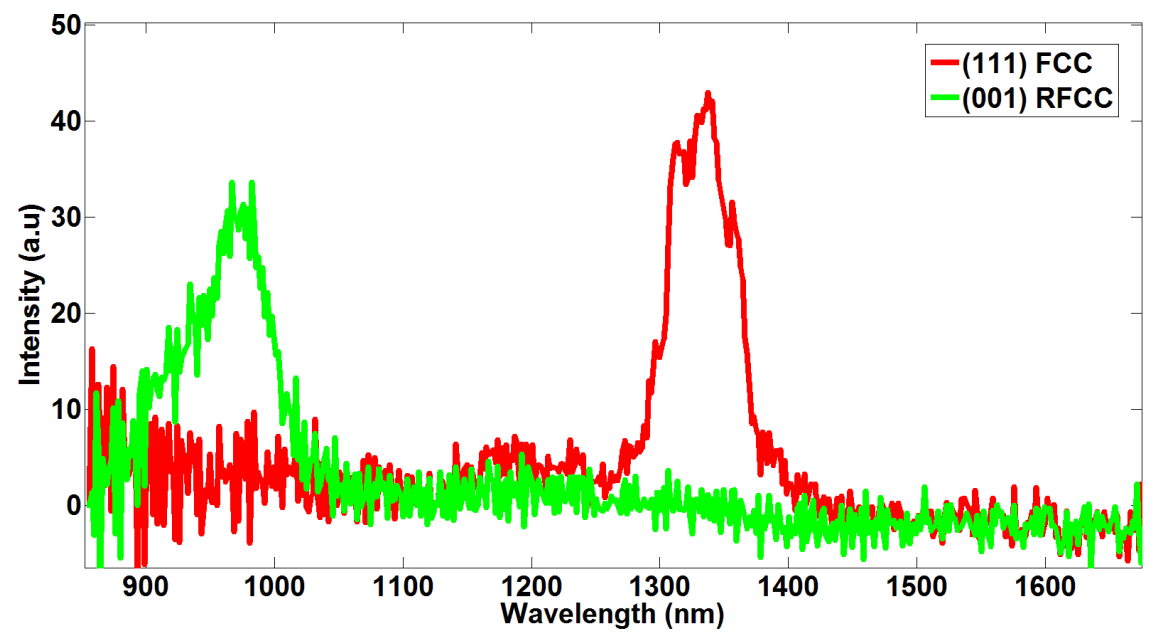

Fig. 13: Reflection on different position of the sedimentated sample.

\section{CONCLUSIONS}

We have simulated different FCC structures that can be integrated into polymer waveguides to show that core-shell colloidal photonic crystal structures can be used for achieving sharp bends in polymer waveguides. Air-clad silica spheres photonic crystal structures can only provide reflections $15 \%$ as compared to more than $50 \%$ reflections from core-shell structures. Truncated core-shell photonic crystal structures on rectangular seed layer are calculated to reflect more than $70 \%$ of the incident light. Different fabrication techniques for fabrication of silica spheres FCC structures on top of rectangular seed layer have been investigated and implemented. Our initial characterization results show that FCC structures with (001) FCC ground plane can be fabricated which can be distinguished from (111) FCC or RFCC structures using optical characterization.

\section{REFERENCES}

[1] M. V. Rybin, A. V. Baryshev, A. B. Khanikaev, M. Inoue, K. B. Samusev, A. V. Sel'kin, G. Yushin, and M. F. Limonov, "Selective manipulation of stop-bands in multi-component photonic crystals: Opals as an example," Phys. Rev. B 77, 205106 (2008).

[2] M. Soljacic, S. G. Johns, S. Fan, M. Ibanescu, E. Ippen and J. D. Joannopoulos, "Photonic-crystal slow-light enhancement of nonlinear phase sensitivity," JOSA B19, p. 2052-2059 (2002).

[3] Y. Ohtera, T. Sato, T. Kawashima, T. Tamamura and S. Kawakami, "Photonic crystal polarisation splitters," Electron. Lett., 35, p.1271-1272 (1999).

[4] O. Toader, S. John, K. Busch, "Optical trapping, Field enhancement and Laser cooling in photonic crystals," Opt. Express 8, p. 217-222 (2001).

[5] M. Soljacic and J. D. Joannopoulos, "Enhancement of nonlinear effects using photonic crystals," nature materials 3, p.211-219 (2004).

[6] A. Mihi, M. Ocana, H. Miquez, "Oriented Colloidal-Crystal Thin Films by Spin-Coating Microspheres Dispersed in Volatile Media," Adv. Materials 18, p.2244-2249 (2006).

[7] H. Yang and P. Jiang, "Large-Scale Colloidal Self-Assembly by Doctor Blade Coating," Langmuir 26, p.1317313182, (2010).

[8] Y. L. Wu, "Control over Colloidal Crystallization by Shear and Electric Fields," PhD Thesis, Utrecht University, (2007).

[9] A. van Blaaderen, R. Ruel, and P. Wilzius, “Template Directed Colloidal Crystallization," Nature 385, 321-324 (1997). 